\title{
Percepção materna do estado nutricional de seus filhos
}

\author{
Mother's perception of their children's \\ nutritional status
}

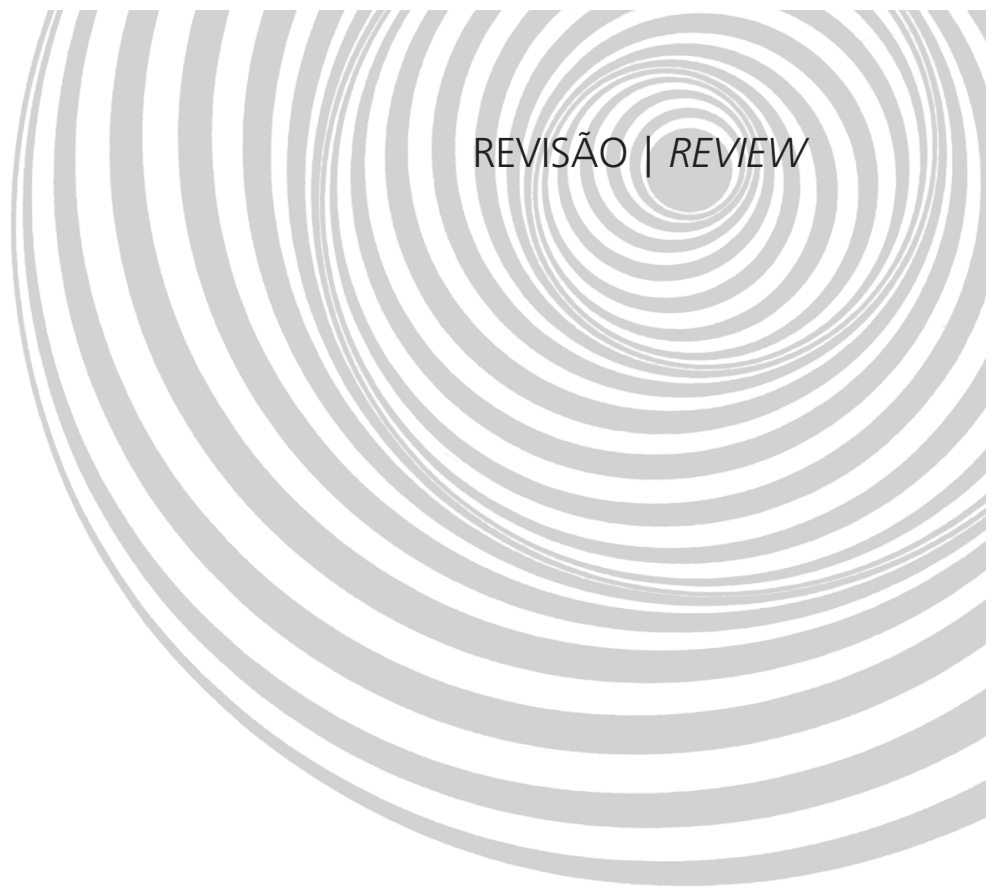

Paula CHUPROSKI ${ }^{1}$

Débora Falleiros de MELLO²

RE S U M O

Este estudo tem por objetivos caracterizar a percepção das mães sobre o estado nutricional de seus filhos e destacar os fatores que interferem nessa percepção, a partir de uma revisão sistemática da literatura. Para a seleção dos artigos foram utilizadas as bases de dados Lilacs e Medline, e a amostra final foi constituída de 17 artigos analisados na íntegra. Os aspectos que emergiram das produções selecionadas são apresentados configurando uma análise descritiva por categorização. Os achados referem-se ao estado nutricional da mãe relacionado ao estado nutricional da criança; aos padrões socioculturais e à percepção do peso da criança; à utilização de gráficos de crescimento por profissionais de saúde e à visão das mães. As mães apresentam dificuldade para reconhecer o peso do seu filho e os fatores que podem influenciar na percepção do peso são: idade da criança, sexo, peso, padrões sociais e culturais e escolaridade materna.

Termos de indexação: Criança. Mães. Percepção de peso.

\section{A B S T R A C T}

This study aims to characterize mother's perception of their children's nutritional status and highlight factors interfering in this perception, based on a systematic literature review. To select the articles, the databases Lilacs and Medline were used, and the final sample consisted of 17 articles whose full version was analyzed. The aspects that emerged from the selected articles are presented, configuring a descriptive analysis by categorization. The findings refer to the mother's nutritional status related to the child's nutritional status; sociocultural standards and perception of the child's weight; use of growth graphs by health professionals and the mothers' view. It is difficult for the mothers to recognize their child's weight and the factors that can influence weight perception are: child's age, gender, weight, social and cultural standards and mother's education level.

Indexing terms: Child. Mothers. Weight perception.

\footnotetext{
1 Universidade Estadual do Centro Oeste, Departamento de Nutrição. Guarapuava, PR, Brasil.

2 Universidade de São Paulo, Escola de Enfermagem, Departamento de Enfermagem Materno Infantil e Saúde Pública. Av. dos Bandeirantes, 3900, Monte Alegre, 14040-902, Ribeirão Preto, SP, Brasil. Correspondência para/Correspondence to: D.F MELLO.E-mail: <defmello@eerp.usp.br>.
} 


\section{N T R O D U ÇÃ O}

As práticas alimentares adotadas na infância têm sido consideradas como inteiramente dependentes dos pais, principalmente das mães, de seus comportamentos, decisões e percepções'.

O desempenho das mães é apontado como chave no desenvolvimento dos comportamentos e preferências alimentares, na regulação da ingestão energética e nos padrões de atividade física das crianças ${ }^{2-4}$.

Atualmente, a população infantil vem passando por um processo de transição nutricional e a obesidade, nessa população, já é considerada endêmica nos países desenvolvidos e vem crescendo rapidamente nos países em desenvolvimento ${ }^{5-7}$. A desnutrição na infância, caracterizada pelo comprometimento acentuado do crescimento linear e/ou pelo emagrecimento extremo da criança, também configura um dos maiores problemas nos países em desenvolvimento8.

Os programas para prevenir ou tratar a obesidade na infância são improváveis de ter sucesso, se as mães não reconhecem as suas crianças como tendo excesso de adiposidade, ou se não reconhecem os riscos associados ao excesso de gordura acumulado $3,9,10$.

A percepção adequada da mãe em relação ao peso de seu filho pode ser o requisito para a procura de uma assistência profissional especializada e a aderência ao tratamento proposto ${ }^{5}$.

A assistência prestada nos serviços de saúde terá um impacto limitado se não se considerar que a mãe, os responsáveis e os cuidadores exercem um papel fundamental na recuperação, na manutenção, na prevenção de doenças e na proteção da saúde da criança no domicílio. Muitas questões relacionadas ao estado nutricional na infância vêm sendo discutidas e analisadas na atualidade, sendo relevante a revisão sistemática da literatura para ampliar as reflexões. Assim, este estudo tem por objetivos caracterizar as percepções das mães sobre o estado nutricional de seus filhos e destacar os fatores que interferem nessa percepção, a partir de uma revisão sistemática da literatura.

\section{MÉTODOS}

Este estudo configura uma revisão sistemática da literatura, caracterizada como um instrumento da prática baseada em evidências, que possibilita a síntese e a análise do conhecimento produzido acerca da temática investigada, constituindo-se em uma técnica com rigor metodológico, aumentando a confiabilidade e a profundidade das conclusões da revisão ${ }^{11,12}$.

As seguintes etapas foram contempladas: a formulação da questão norteadora e dos objetivos da revisão, o estabelecimento de critérios de inclusão e exclusão dos artigos, a definição dos dados a serem coletados, a análise dos resultados, a discussão e a apresentação dos resultados. A questão norteadora adotada para esse estudo foi: as mães percebem o estado nutricional de seus filhos?

Para a seleção dos artigos foram utilizadas as palavras-chave: percepção, mãe, criança e peso, junto às bases de dados Lilacs e Medline. Foram incluídos artigos publicados em português, inglês e espanhol, com os resumos disponíveis nas bases de dados descritas, no período compreendido entre os anos de 1998 e 2007, que abordassem a temática da percepção materna em relação ao estado nutricional da criança.

Foram consideradas publicações com todos os tipos de delineamentos e evidências científicas, devido à característica da questão norteadora, que não se relacionaram à eficácia de uma intervenção, mas sim aos principais fatores que interferiram na percepção das mães sobre o peso da criança. A busca foi realizada pelo acesso on-line e a amostra final foi constituída de 17 artigos.

Para a análise dos resultados e a posterior síntese dos artigos analisados na íntegra, que atenderam aos critérios de inclusão, foi utilizada uma tabela com título, autores, metodologia, resultados e recomendações/conclusões.

A seleção dos estudos, a partir da busca on-line, e a coleta dos dados foram realizadas por dois pesquisadores e confrontadas posteriormente, com vistas a diminuir vieses e garantir 
a qualidade da revisão. Os resultados da revisão estão apresentados de forma descritiva, com a categorização de grupos temáticos.

\section{RESULTADOSE DISCUSSÃO}

Entre os artigos incluídos nesta revisão, três têm como área de atuação a Medicina, dois a Psicologia, dois a Enfermagem, dois a Medicina em conjunto com a Nutrição, um a Nutrição e em sete não foi possível identificar a profissão dos autores.

Quanto ao país de origem dos estudos, sete trabalhos foram desenvolvidos nos Estados Unidos, três no Brasil, dois no Chile, dois na Itália, um na Austrália, um na Argentina e um na Alemanha.

Com relação ao tipo de delineamento dos estudos avaliados, a maioria foi de estudos transversais, que utilizaram questão fechada com escolha de itens, como uma escala de Likert, e dois artigos utilizaram escala de silhuetas do corpo. A síntese do material incluído nesta revisão está no Anexo 1.

Na análise dos dados emergiram as temáticas: estado nutricional da mãe, estado nutricional da criança e escolaridade materna; padrões socioculturais e percepção do peso da criança; utilização de gráficos de crescimento por profissionais de saúde e a visão das mães.

\section{Estado nutricional da mãe e da criança e escolaridade materna}

Alguns estudos apontaram certa distorção da percepção das mães do peso corporal das crianças, que variou de $87,5 \%$ a $24,7 \%$ 9,5; porém, quando foi empregada a escala de silhuetas essa distorção apresentou-se reduzida $(20 \%)^{13}$. Lazzeri et al..$^{13}$ notaram uma elevada subestimação do excesso de peso e da obesidade das crianças, quando foi utilizada uma questão (Em sua opinião a sua criança é: muito magra, um pouco magra, normal, um pouco gorda, gorda ou muito gorda), e isso não ocorreu com a escala de silhuetas. Uma hipótese levantada no estudo foi que as mães podem se sentir desconfortáveis quando respondem uma questão na qual devem classificar seu filho dentro das categorias "gordo ou muito gordo", devido aos fatores emocionais que podem interferir durante o processo de avaliação.

A idade da criança pareceu interferir na percepção do estado nutricional, pois, em alguns estudos, a percepção materna em relação ao peso da criança tornou-se mais evidente com o aumento da idade da criança $a^{1,2,10}$ e do valor do Índice de Massa Corporal $(\mathrm{IMC})^{10}$.

O sexo da criança, em alguns trabalhos, influenciou a percepção materna do peso $0^{10,14}$ e o monitoramento da ingestão alimentar ${ }^{15}$. As mães eram, aproximadamente, três vezes mais prováveis de classificar as meninas em risco de excesso de peso do que os meninos ${ }^{10}$. Foi apontado que as mães estavam mais preocupadas com o ganho de peso excessivo nas suas filhas e que o monitoramento da ingestão alimentar era previsto para a filha, independentemente do estado do peso, quando a própria mãe fazia restrição dietética ${ }^{15}$.

Maynard et al. ${ }^{10}$ e Díaz ${ }^{16}$ apontaram que as mães de crianças com excesso de peso apresentavam valores de IMC mais elevados, quando comparadas às mães de crianças com um estado de peso menor, assim como existiu uma correlação entre o IMC materno e o IMC da criança ${ }^{14}$. Um estudo ${ }^{17}$ destacou que a probabilidade de a criança apresentar excesso de peso foi maior nas mães com obesidade, outro ${ }^{2}$ mostrou que metade das mães com sobrepeso ou obesidade tinha uma criança com excesso de peso. Há algumas décadas esse fato tem sido elucidado na literatura, com demonstração de que se um dos pais é obeso a chance de a criança desenvolver obesidade é de $40 \%$, mas, se ambos os pais são obesos essa chance pode ser de até $80 \%{ }^{18,19}$.

Quanto ao nível educacional e ao IMC maternos, relacionados ao IMC da criança, alguns estudos ${ }^{2,13,20}$ destacaram que um alto nível de educação correspondeu à menor porcentagem de obesidade em mães e crianças. 
O nível de educação materna e a percepção do estado nutricional da criança foram investigados $2,13,16,20$, entretanto houve uma divergência. Alguns apontaram que níveis de educação materna menores estavam associados a falhas na percepção do excesso de peso de $\operatorname{crianças}^{20}$, e um nível educacional mais alto ajudou as mães a perceber melhor o peso da criança e, aparentemente, aplicar estratégias de controle da ingestão alimentar ${ }^{2}$. Outros trabalhos ${ }^{13,16}$ não encontraram diferenças significantes entre o nível educacional da mãe e a percepção do peso da criança.

\section{Padrões socioculturais e percepção do peso da criança}

As questões sociais e culturais também foram levantadas em estudos desta revisão, apontando aspectos do estado nutricional da criança e sua percepção pela mãe.

Alguns trabalhos destacaram a percepção materna do peso da criança ligada ao padrão sociocultural estereotipado da criança considerada gorda como bela e sadia, em algumas sociedades $^{5,16,21}$, e a crença de que essa criança estaria recebendo um melhor cuidado dos pais 5 .

Em alguns artigos foi verificado que, apesar de não reconhecerem o estado nutricional da criança, as mães de crianças obesas acreditavam que era mais saudável uma criança com peso normal, tinham consciência das enfermidades relacionadas à obesidade ${ }^{16}$ e expressavam preocupação com a saúde e o peso futuro da criança2,4,22. As mães que relatavam essa preocupação eram mais prováveis de restringir a ingestão de alimentos da criança e menos prováveis de pressionar a criança a comer, porém não foi detectada uma associação entre as estratégias alimentares e as crianças com excesso de peso ${ }^{4}$.

Em um estudo ${ }^{22}$ que avaliou as práticas alimentares das mães entre irmãos obesos e não obesos, também foi verificado que o controle materno sobre a alimentação das crianças não estava relacionado ao peso das mesmas.
As crenças e as práticas culturais com relação ao excesso de peso, à educação e à alimentação da criança podem se diferenciar entre os grupos étnicos ${ }^{23}$.

As práticas alimentares compreendem processos sociais e culturais que comportam valores, identidades, aprendizagem, escolhas e gostos alimentares, conformados no modo de vida e permeados por várias ambigüidades, que denotam simultaneamente tradições e mudanças ${ }^{24}$.

Um trabalho ${ }^{25}$ que buscou apreender a percepção das mães sobre a perda de peso e a desnutrição da criança, identificando os valores, mitos, modos de cuidar e/ou buscar cuidado, identificou que os pensamentos das mães em relação à desnutrição estavam associados aos prejuízos imediatos ao filho e não como causa e conseqüência das condições sociais e econômicas a que estavam expostas.

\section{Uso de gráficos de crescimento por profissionais de saúde e a visão das mães}

Os gráficos de crescimento podem não ser significativos ou compreensíveis por certas populações, pois os pais podem considerar o local que a criança ocupa no gráfico como um sinal de competência deles. Se os pais consideram um tamanho grande desejável para a criança, o uso dos gráficos de crescimento pelos profissionais de saúde para indicar excesso de peso pode confundir os pais e não enfatizar a relevância dos gráficos ${ }^{3}$.

Os artigos tentaram explicar a distorção da percepção materna do peso da criança ${ }^{2,3,23}$, e apontaram a existência de diferenças entre como os pais e os profissionais de saúde percebiam a definição, a etiologia e o tratamento do excesso de peso em crianças $^{3}$, que mães e pediatras não tinham o mesmo ponto de vista com relação ao problema da obesidade na infância ${ }^{2}$, que as mães apresentavam dificuldade de entender os gráficos de crescimento $^{9}$, e, ainda, que não achavam significativo o uso desses para definir a criança como tendo excesso de peso ${ }^{3}$. 
Um estudo qualitativo ${ }^{3}$ buscou apreender as percepções de mães de baixo rendimento econômico que tinham uma criança com excesso de peso, e encontrou que as mães consideravam a criança como tendo um peso saudável, enquanto as funções sociais e de atividade física da criança não estivessem afetadas.

\section{O N CLUS Ã O}

Nesta revisão sistemática foi encontrado que o estado nutricional das crianças está relacionado ao estado nutricional das mães, o que já vem sendo apontado pela literatura há algum tempo.

Dos estudos analisados, as mães apresentaram dificuldades para reconhecer o peso do seu filho, apesar de demonstrarem preocupação com o peso futuro da criança e de considerarem mais saudável uma criança com peso normal. A percepção materna do peso da criança pode ser influenciada pela idade da criança, pelo sexo, pelo peso, pelos padrões socioculturais e pela escolaridade materna.

Os profissionais de saúde que trabalham com crianças e famílias necessitam repensar as práticas de saúde, procurando focar o entendimento e as habilidades das mães, uma vez que elas podem não compreender o significado do lugar que seu filho ocupa no gráfico de crescimento.

No cuidado à saúde da criança é relevante tematizar conceitos, situações de saúde, as particularidades e os elementos culturais das famílias, a organização da assistência, o próprio encontro e a interação entre os sujeitos, buscando contribuir para a reconstrução das práticas de saúde.

\section{O LABORADORES}

P. CHUPROSKI colaborou com a elaboração do trabalho, participou de todas as etapas da pesquisa e da redação do artigo. D.F. MELLO idealizou o projeto e participou da análise e da redação do artigo.

\section{REFERÊ NCIAS}

1. Kroke A, Strathmann S, Günther ALB. Maternal perceptions of her child's body weight in infancy and early childhood and their relation to body weight status at age 7. Eur J Pediatr. 2006:165 (12):875-83. doi: 10.1007/s00431-006-0191-3.

2. Genovesi S, Giussani M, Faini A, Vigorita F, Pieruzzi F, Strepparava MG, et al. Maternal perception of excess weight in children: a survey conducted by paediatricians in the province of Milan. Acta Paediatr. 2005; 94(6):747-52. doi: 10.1080/080 35250510028894

3. Jain A, Sherman SN, Chamberlin LA, Carter Y, Power SW, Whitaker RC. Why don't low-income mothers worry about their preschoolers being overweight? Pediatrics. 2001; 107(5):1138-46.

4. May AL, Donohue M, Scanlon KS, Sherry B, Dalenius K, Faulkner $\mathrm{P}$, et al. Child-feeding strategies are associated with maternal concern about children becoming overweight, but not children's weight status. J Am Diet Assoc. 2007; 107(7):1167-74. doi: 10.1016/j.jada.2007.04.009.

5. Boa-Sorte N, Neri AL, Leite MEQ, Brito SM, Meirelles AR, Luduvice FBS, et al. Percepção materna e autopercepção do estado nutricional de crianças e adolescentes de escolas privadas. J Pediatr. 2007; 83(4):349-56. doi: 10.2223/JPED.1678.

6. Brasil. Ministério da Saúde. Secretaria de Atenção à Saúde. Guia alimentar para a população brasileira: promovendo a alimentação saudável. Brasília: Ministério da Saúde; 2005.

7. Brasil. Ministério da Saúde. Secretaria de Política de Saúde. Guia alimentar para crianças menores de dois anos. Brasília: Ministério da Saúde; 2002.

8. Monteiro CA, Conde WL. Tendência secular da desnutrição e da obesidade na infância na cidade de São Paulo (1974-1996). Rev Saúde Pública. 2000; 34(6):52-61. doi: 10.1590/S0034-891020 00000700008

9. Hirschler V, González C, Cemente G, Talgham S, Petticnichio $\mathrm{H}$, Jadzinsky M. ¿Cómo perciben las madres de niños de jardín de infantes a sus hijos con sobrepeso? Arch Argent Pediatr. 2006; 104(3): 221-6.

10. Maynard LM, Galuska DA, Blanck HM, Serdula MK. Maternal perceptions of weight status of children. Pediatrics. 2003; 111(5):1226-31. doi: 10.1542/ peds.111.5.S1.1226.

11. Dickson R. Systematic reviews. In: Hamer S, Collison G. Achieving evidence-based practice: a handbook for practioners. London: Ballière Tindall; 1999. p.41-60. 
934 | P. CHUPROSKI \& D.F. MELLO

12. Galvão CM, Sawada NO, Trevizan MA. Revisão sistemática: recurso que proporciona a incorporação das evidências na prática da enfermagem. Rev Latino Am Enfermagem. 2004; 12(3):549-56. doi: 10.1590/S0104-11692004000300014.

13. Lazzeri G, Casorelli A, Giallombar D, Grasso A, Guidoni C, Menoni E, et al. Nutritional surveillance in Tuscany: maternal perception of nutritional status of 8-9 y-old school-children. J Prev Med Hyg. 2006; 47(1):16-21.

14. Olvera N, Suminski R, Power TG. Intergenerational perceptions of body image in hispanics: role of $\mathrm{BMI}$, gender, and acculturation. Obes Res. 2005; 13(11):1970-9. doi: 10.1038/oby.2005.242.

15. Tiggemann M, Lowes J. Predictors of maternal control over children's eating behaviour. Appetite. 2002; 39(1)1-7. doi: 10.1006/appe.2002.0487.

16. Díaz M. Percepción materna del estado nutritivo de sus hijos obesos. Rev Chil Pediatr. 2000; 71(4): 316-20. doi: 10.4067/S0370-4106200000040 0006.

17. Bracho F, Ramos E. Percepción materna del estado nutricional de sus hijos: ¿Es un factor de riesgo para presentar malnutrición por exceso? Rev Chil Pediatr. 2007; 78(1):20-7. doi: 10.4067/S037041062007000100003.

18. American Academy of Pediatrics. Committee on Nutrition. Prevention of pediatric overweight and obesity. Pediatrics. 2003;112(2):424-30.
19. Vitolo MR. Nutrição: da gestação à adolescência. Rio de Janeiro: Reichmann \& Affonso Editores; 2003.

20. Baughcum AE, Chamberlin LA, Deeks CM, Power SW, Whitaker RC. Maternal perceptions of overweight preschool children. Pediatrics. 2000; 106(6):1380-6. doi: 10.1542/peds.106.6.1380.

21. Araújo MFM, Lemos ACS, Chaves ES. Creche comunitária: um cenário para a detecção da obesidade infantil. Ciên Cuidados Saúde. 2006; 5(1):24-31.

22. Saelens BE, Ernst MM, Epstein LH. Maternal child feeding practices and obesity: a discordant sibling analysis. Int J Eat Disord. 2000; 27(4):459-63. doi: 10.1002/(SICI)1098-108X(200005)27:4.

23. Kasemsup $R$, Reicks $M$. The relationship between maternal child-feeding practices and overweight in Hmong preschool children. Ethn Dis. 2006; 16(1):187-93.

24. Canesqui AM, Garcia RWD. Uma introdução à reflexão sobre a abordagem sociocultural da alimentação. In: Antropologia e nutrição: um diálogo possível. Rio de Janeiro: Fiocruz; 2005. p.9-19.

25. Lopes MSV, Machado MFAS, Vieira NFC. Percepção das mães sobre a perda de peso e desnutrição dos filhos. Nursing. 2003; 57(6):30-4.

Recebido em: 7/3/2008

Versão final reapresentada em: 2/8/2009 Aprovado em: 23/10/2009 


\section{ANEXO 1}

SÍNTESE DOS ARTIGOS INCLUÍDOS NA REVISÃO SISTEMÁTICA SOBRE A PERCEPÇÃO DAS MÃES

QUANTO AO ESTADO NUTRICIONAL DE SEUS FILHOS

\begin{abstract}
Metodologia e Resultados
Aplicação de questionários e avaliação antropométrica dos pais e crianças.

O controle materno da alimentação das crianças avaliado pelo Child-Feeding Questionnaire foi similar para os irmãos obesos e não obesos 22 .

Estudo transversal com aplicação de um questionário às mães para avaliar a percepção do estado nutricional de seus filhos obesos e os conhecimentos gerais sobre obesidade. $64,1 \%$ das mães apresentaram uma percepção do peso distorcida e $98,4 \%$ acreditaram que é mais saudável uma criança com peso normal ${ }^{16}$.
\end{abstract}

Estudo transversal usando um questionário que avaliou as práticas alimentares maternas. Avaliação antropométrica das crianças e índice de massa corporal materno.

A maioria das mães demonstrou uma percepção exata do seu estado de peso, entretanto, somente 1 em 5 mães identificou corretamente a sua criança como tendo excesso de peso ${ }^{20}$.

Pesquisa qualitativa (grupos focais) com questões desenvolvidas para explorar a definição, a etiologia e o tratamento do excesso de peso.

As mães não achavam significativo o uso dos gráficos de crescimento para definir o excesso de peso. As mães e os profissionais de saúde viam a definição, a etiologia e o tratamento do excesso de peso de forma distinta ${ }^{3}$.

Aplicação de questionários e avaliação antropométrica das crianças e do índice de massa corporal materno. As mães percebiam as crianças como tendo um peso normal. O estado do peso das crianças estava correlacionado com o monitoramento materno da alimentação ${ }^{15}$.

Abordagem qualitativa. Técnicas: observação participante e entrevista aberta.As mães viam a desnutrição no que essa podia trazer de prejuízos imediatos ao filho e não como causa e conseqüência das condições sociais e econômicas a que estavam expostas $^{25}$.

Foram utilizados dados do Third National Health and Nutrition Examination Survey (NHANES III), associados a dados atuais da percepção do peso e da avaliação antropométrica da criança. Um terço das mães com uma criança com excesso de peso classificou a criança de forma incorreta ${ }^{10}$.

Aplicação de questionário e avaliação antropométrica das mães e crianças. A percepção incorreta do peso da criança foi notada em $37,5 \%$ das mães e foi mais presente em classes educacionais menores. Um nível de educação mais alto pareceu ajudar as mães a aplicar estratégias de controle da ingestão alimentar².

Estudo longitudinal com aplicação de questionários, instrumento ilustrativo para avaliar a percepção do tamanho corporal e avaliação antropométrica das mães e crianças durante quatro anos do estudo.

A percepção das mães do tamanho exato da criança estava significativamente e positivamente correlacionada com o índice de massa corporal da criança ${ }^{14}$.
Recomendações e Conclusão

O impacto da história de peso e dos hábitos alimentares maternos na impressão do peso futuro e da saúde das crianças, independentemente do estado de peso da criança.

Desenvolvimento de estratégias que incluam previamente a definição da percepção materna do estado nutricional ao iniciar um tratamento para obesidade.

Pesquisa futura é necessária para entender a percepção errônea das mães.

A obesidade foi mais comum em mães com menores níveis educacionais, e em suas crianças, assim como a classificação errônea do peso da criança.

Reavaliação de como esses gráficos de crescimento deveriam ser usados em orientações para prevenir a obesidade.

A melhor aproximação para prevenir a obesidade pode ser focar mais no aumento das habilidades dos pais e menos na discussão do crescimento da criança.

Pesquisas futuras que avaliem as trocas verbais entre os pais e as crianças sobre os tópicos referentes à alimentação, observações de comportamentos e episódios durante as refeições.

É preciso tentar conhecer o cuidado cultural e não deixar o conhecimento centrado somente na visão biomédica.

Necessidade de obter um entendimento mais profundo de como as mães percebem o estado do peso da criança. Programas de intervenção para crianças com excesso de peso são improváveis de ter sucesso se as mães não reconhecem as suas crianças como tendo excesso de adiposidade.

Programas de intervenção para crianças com excesso de peso são improváveis de ter sucesso sem um apoio aos pais. Os pediatras deveriam entender como as mães percebem o problema da obesidade na infância.

Realização de outros estudos que considerem variações no rendimento ou classe social, nível de aculturação, classificação de IMC e outros grupos étnicos. 
Aplicação do Child-Feeding Questionnaire, revisado, para mães da etnia Hmong, divididas em dois grupos: mães de crianças com peso normal e mães de crianças com excesso de peso. Aproximadamente $90 \%$ das mães de crianças com peso normal relataram que a criança estava com o peso normal, enquanto somente $23 \%$ das mães de crianças com excesso de peso relataram que a criança estava com excesso de peso.

Não foi observada relação direta entre as práticas alimentares maternas e o estado de excesso de peso das crianças $^{23}$.

Estudo transversal utilizando escala de silhuetas e questão fechada para avaliar a percepção materna do peso da criança. Avaliação antropométrica das crianças e IMC materno. Houve uma correlação entre a figura escolhida pela mãe e a classe do IMC da criança em $80 \%$. Em relação à questão fechada, existiu uma elevada subestimação do excesso de peso e da obesidade da criança $^{13}$.

Estudo transversal com abordagem quantitativa-descritiva. As mães de crianças com sobrepeso ou obesidade responderam questões sobre percepção do estado nutricional da criança, período de amamentação e situação socioeconômica das famílias.76\% das mães tinham uma percepção incorreta do peso da criança.60\% das crianças com excesso de peso apresentavam um padrão de amamentação ineficaz, segundo critérios da Organização Mundial da Saúde ${ }^{21}$.

Estudo transversal a partir da avaliação nutricional das crianças e da aplicação de questionário às mães sobre a percepção dos hábitos alimentares e da imagem corporal da criança. Diferença significativa na freqüência de distorção da percepção da imagem corporal entre as mães de crianças eutróficas e as mães de crianças que apresentavam risco de sobrepeso ou sobrepeso (17\% contra $87,5 \%)^{9}$.

Foram utilizados dados do estudo longitudinal DONALD.

A percepção materna do peso da criança foi avaliada aos 6 meses, aos 12 meses, aos 2 anos e aos 4 anos, e foi verificado se essa percepção interferiu no estado do peso da criança aos 7 anos de idade.

A avaliação da percepção materna ao longo tempo e o desenvolvimento do peso nas crianças mostrou que, as consideradas muito abaixo do peso ganharam mais peso até os 7 anos, quando comparadas as com as de peso normal. Crianças consideradas muito acima do peso perderam mais peso até os 7 anos, quando comparadas às com peso normal'.

Estudo descritivo de corte transversal com aplicação de questionário e avaliação antropométrica das crianças e das mães.

A percepção do estado nutricional das crianças foi adequada em $54,07 \%$ das mães, porém $41,48 \%$ delas tendiam a subestimar o excesso de peso da criança ${ }^{17}$.

Estudo transversal utilizando 6 frases do Child Feeding Questionnaire. Avaliação antropométrica das crianças.

A percepção do estado nutricional das crianças foi adequada, somente, em $21 \%$ das mães que tinham uma criança com excesso de peso.

A percepção materna da criança como acima do peso não estava associada a estratégias de restrição ou pressão alimentar ${ }^{4}$.

Estudo transversal, com avaliação do estado nutricional das crianças e dos adolescentes e aplicação de questionário às mães, às crianças e aos adolescentes. Em relação à percepção do estado nutricional, 75,3\% das mães tiveram uma percepção correta do peso da criança, $18,4 \%$ subestimaram o peso e $6,3 \%$ superestimaram o estado nutricional ${ }^{5}$.
A falta de relação entre as práticas alimentares maternas e o estado de peso da criança pode ser devido a fatores como educação da mãe, papel de outros cuidadores e fatores culturais.

A escala de silhuetas precisa ser aperfeiçoada para aumentar a sua exatidão antes de ser regularmente utilizada em investigações epidemiológicas, já que houve uma variação de $20 \%$ nos resultados.

A percepção materna inadequada do estado nutricional do filho é uma problemática na dinâmica familiar que possibilita a gênese da obesidade infantil.

Centros especializados deveriam desenvolver trabalhos para avaliar a atividade física e a qualidade da dieta, que não por um questionário.

A percepção das mães sobre a imagem e os hábitos alimentares predispõe o sobrepeso.

Um melhor entendimento das influências da percepção materna de peso é necessário.

A percepção materna do peso pode ser um importante determinante do desenvolvimento do peso da criança.

A alteração da percepção materna do estado nutricional das crianças é um fator importante a ser analisado para tentar diminuir a alta prevalência de excesso de peso, e, assim, evitar as conseqüências deletérias associadas a esse estado.

As mães preocupadas com a possibilidade de a criança ter excesso de peso eram mais prováveis de restringir a ingestão alimentar das crianças e menos prováveis de pressionar a criança a comer.

A falta de percepção adequada do peso ocorreu com muita freqüência entre as crianças e suas genitoras, em especial quando havia excesso de peso, fatores que podem representar obstáculos ao correto reconhecimento das alterações nutricionais. 